\title{
A computational investigation of the ventilation structure and maximum rate of metabolism for a physiologically based pharmacokinetic (PBPK) model of inhaled xylene
}

\author{
Karen A. Yokley* ${ }^{*}$, Jaclyn Ashcraft*, and Nicholas S. Luke ${ }^{\dagger}$ \\ ${ }^{*}$ Department of Mathematics and Statistics \\ Elon University, Elon, NC 27244 \\ ${ }^{\dagger}$ Department of Mathematics \\ North Carolina A\&T State University, Greensboro, NC 27411 \\ $\ddagger$ Corresponding Author: kyokley@elon.edu
}

Received: 29 August 2018, accepted: 6 January 2019, published: 4 February 2019

\begin{abstract}
Physiologically based pharmacokinetic (PBPK) models are systems of ordinary differential equations that estimate internal doses following exposure to toxicants. Most PBPK models use standard equations to describe inhalation and concentrations in blood. This study extends previous work investigating the effect of the structure of air and blood concentration equations on PBPK predictions. The current study uses an existing PBPK model of xylene to investigate if different values for the maximum rate of toxicant metabolism, $V_{\max }^{x y l}$, can result in similar compartmental predictions when used with different equations describing inhalation. Simulations are performed using $V_{\max }^{x y l}$ values based on existing literature. Simulated data is also used to determine specific $V_{\max }^{x y l}$ values that result in similar predictions from different ventilation structures.
\end{abstract}

Differences in ventilation equation structure may affect parameter estimates found through inverse problems, although further investigation is needed with more complicated models.

Keywords-PBPK modeling, xylene

\section{INTRODUCTION}

Physiologically based pharmacokinetic (PBPK) modeling uses ordinary differential equations to describe absorption, distribution, metabolism, and excretion of toxicants following exposure. PBPK models have been developed to estimate internal doses for various toxicants in rodents [1] [9] [11] [14] [17] [18] [25] [34] [37] [40] and in humans [2] [3] [8] [10] [13] [15] [23] [26] [35] [36] [39] [38] [43] [48]. Most PBPK model equations use

Copyright: (C) 2019 Yokley et al. This article is distributed under the terms of the Creative Commons Attribution License (CC BY 4.0), which permits unrestricted use, distribution, and reproduction in any medium, provided the original author and source are credited.

Citation: Karen A. Yokley, Jaclyn Ashcraft, Nicholas S. Luke, A computational investigation of the ventilation structure and maximum rate of metabolism for a physiologically based pharmacokinetic (PBPK) model of inhaled xylene, Biomath 8 (2019), 1901067, http://dx.doi.org/10.11145/j.biomath.2019.01.067 
the same basic structure and assumptions, such as the assumption that compartments are well-mixed and that the transfer of some chemicals is at equilibrium. However, the appropriate use of PBPK model estimates in risk assessment is dependent on multiple factors, including the biological basis of the model parameters and structure [6].

The industrial solvent xylene is a component of paints, paint thinners, and related products [42]. Workers in specific industries may be at higher risk for xylene exposure and poorly ventilated areas may amplify exposure dangers [16], and xylene may increase the effect of other chemicals when present in mixtures [4]. As in [46], the PBPK model of xylene from [39] is used for the current investigation because of the relative simplicity of the model.

The purpose of this project is to expand on the previous PBPK investigation that considered different modeling approaches for ventilation [46] and to also consider variation or errors in the parameter for the maximum rate of metabolism, $V_{\max }(\mathrm{mg} / \mathrm{h})$. Yokley [46] considered three structures for modeling ventilation, described as "equilibrium," "non-equilibrium," and "hybrid" models. The results were very similar for the equilibrium and hybrid models, and therefore only two structures will be used to model ventilation in the current study. The equilibrium model uses the standard quotient for the concentration of toxicant in arterial blood, and the non-equilibrium model allows for a separate lung compartment. The equilibrium and non-equilibrium models are used to predict various xylene concentrations and amounts in the body following inhalation exposure using different values for the maximum rate of metabolism in the liver. Results of simulations with different metabolic rates are then used in inverse problems to investigate the success of optimization using different types of data.

\section{Model InVEstigation}

\section{A. Model Background}

In [46], a PBPK model for xylene exposure in humans from [39] was used with different

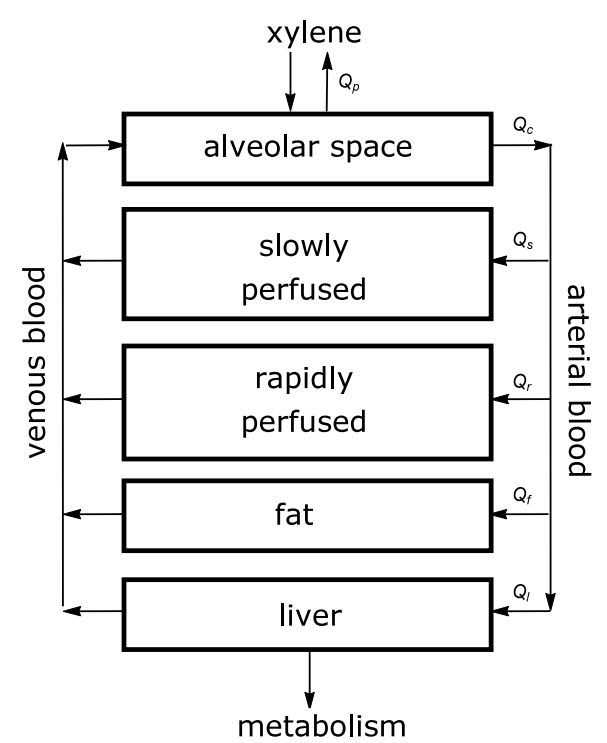

Fig. 1. The structure of the PBPK model of xylene from Tardif et al. [39] used in [46].

equations describing ventilation exposure in order to determine the effect of the structure of those equations on model output. The four compartment model described xylene concentration in the slowly perfused tissues, the rapidly perfused tissues, the fat, and the liver. A schematic of the overall model is presented in Figure 11. For easy reference, a summary of the notations used throughout the model is presented in the Appendix.

The amount of xylene within each compartment depicted in Figure 1 is modeled by a separate differential equation. Additional equations are added to the model to incorporate the ventilation structure. For each model equation, the compartmental concentration is defined as

$$
C_{j}^{x y l}=\frac{A_{j}^{x y l}}{V_{j}},
$$

where $C_{j}^{x y l}$ represents the concentration of the 
chemical, xylene, in compartment $j(m g / L) ; A_{j}^{x y l}$ represents the amount of the chemical, xylene, in compartment $j(m g)$; and $V_{j}$ represents the volume of compartment $j(L)$. Compartment $j$ is either the slowly perfused tissue (denoted $s$ ), the rapidly perfused tissue (denoted $r$ ), the adipose tissue or fat (denoted $f$ ), or the liver (denoted $l$ ).

The differential equation for the change in the amount of xylene within each internal compartment $j$ (excluding the liver) takes the form

$$
\frac{d A_{j}^{x y l}}{d t}=Q_{j}\left(C_{a r t}^{x y l}-\frac{C_{j}^{x y l}}{P_{j}^{x y l}}\right),
$$

where $Q_{j}$ represents the rate of blood flow to compartment $j(L / h) ; C_{\text {art }}^{x y l}$ is the concentration of the chemcial, xylene, in the arterial blood $(m g / L)$; and $P_{j}^{x y l}$ is the partition coefficient for xylene in tissue $j$ (unitless).

The differential equation representing the change in the amount of xylene within the liver takes a different form because xylene is metabolized in the liver. The structure of the equation is similar to that of the other compartments, with a basic flow in minus flow out, but an additional term is subtracted to represent the metabolism. The form of the equation for the liver is

$$
\frac{d A_{l}^{x y l}}{d t}=Q_{l}\left(C_{a r t}^{x y l}-\frac{C_{l}^{x y l}}{P_{l}^{x y l}}\right)-\frac{V_{m a x}^{x y l} \frac{C_{l}^{x y l}}{P_{l}^{x y l}}}{K_{m}^{x y l}+\frac{C_{l}^{x y l}}{P_{l}^{x y l}}},
$$

where $V_{\max }^{x y l}$ is the maximum rate of metabolism for xylene $(m g / h)$ and $K_{M}^{x y l}$ is the concentration of xylene at half saturation $(\mathrm{mg} / \mathrm{L})$.

The full PBPK model is comprised of three equations of form (2) (one each for the slowly perfused, rapidly perfused, and fat compartments), equation (3), and two or more equations that represent the ventilation structures, which are outlined below.

The three ventilation structures used in [46] were classified as "equilibrium," "nonequilibrium," and "hybrid" cases. Many PBPK models use the "equilibrium" case equations for blood concentrations [14] [23] [39], which are constructed under a few assumptions including that the amount of toxicant leaving the alveolar space is equal to the amount eliminated through the blood. Equations similar to (4) and (5) below are typically used in PBPK modeling for concentrations of a particular toxicant, $i$, in the venous (ven) and arterial (art) blood:

$$
\begin{aligned}
C_{\text {art }}^{i}= & \frac{Q_{c} C_{v e n}^{i}+Q_{p} C_{i n h}^{i}}{Q_{c}+\frac{Q_{p}}{P_{b l: a i r}^{i}}} \\
C_{\text {ven }}^{i}= & \frac{\sum_{j} Q_{j} \frac{A_{j}^{i}}{P_{j}^{i} V_{j}}}{Q_{c}},
\end{aligned}
$$

where $Q_{c}$ represents the cardiac flow rate, defined as the sum of the compartmental flow rates $(L / h)$; $Q_{p}$ is the pulmonary flow or alveolar ventilation $(L / h)$; and $P_{b l: a i r}$ is the blood/air partition coefficient (unitless).

The "non-equilibrium" case allows for the amount of toxicant leaving the alveolar space (alv) to not equal the amount entering the alveolar space and thus the alveolar space, arterial concentration, and venous concentration are each represented with their own compartment in the model. This scenario can be modeled using the equations

$$
\begin{aligned}
\frac{d A_{a l v}^{i}}{d t}= & Q_{p}\left(C_{\text {inh }}^{i}-\frac{A_{\text {alv }}^{i}}{V_{\text {alv }} P_{\text {bl:air }}^{i}}\right) \\
& +Q_{c}\left(C_{\text {ven }}^{i}-\frac{A_{\text {alv }}^{i}}{V_{\text {alv }} P_{\text {alv:air }}^{i}}\right) \\
\frac{d C_{a r t}^{i}}{d t}= & Q_{c}\left(\frac{A_{\text {alv }}^{i}}{V_{\text {alv }} P_{\text {alv }}^{i}}-C_{\text {art }}^{i}\right) \\
\frac{d C_{\text {ven }}^{i}}{d t}= & \frac{1}{V_{\text {ven }}}\left(\sum_{j} Q_{j} \frac{A_{j}^{i}}{P_{j}^{i} V_{j}}-Q_{c} C_{v e n}^{i}\right)
\end{aligned}
$$

which are described more fully in [46]. The "hybrid" case involves using the "non-equilibrium" equations (6)-(8) with the alveolar partition coefficient, $P_{a l v}^{i}$, set to be 1 . Because the results from [46] were very similar between the "equilibrium" and "hybrid" cases, the "hybrid" scenario is omitted from the current investigation.

\section{B. Fixed Parameter Values}

All simulations use a body weight $B W$ of $70 \mathrm{~kg}$, under the assumption that $1 \mathrm{~L}=1 \mathrm{~kg}$ 
K.A. Yokley, J. Ashcraft, N.S. Luke, A computational investigation of the ventilation structure and ...

TABLE I

THE COMPARTMENTAL FLOWS AND PARTITION COEFFICIENTS USED IN SIMULATIONS OF THE XYLENE PBPK MODEL.

\begin{tabular}{|l|r|r|}
\hline Compartmental flow & Value $\left(\% Q_{c}\right)$ & Source \\
\hline Fat & 5 & {$[39]$} \\
Slowly perfused & 25 & {$[39]$} \\
Richly perfused & 44 & {$[39]$} \\
Liver & 26 & {$[39]$} \\
\hline Partition coefficient & Value & Source \\
\hline Blood:air & 26.4 & {$[39]$} \\
Fat:blood & 77.8 & {$[39]$} \\
Slowly perfused:blood & 3.0 & {$[39]$} \\
Richly perfused:blood & 4.42 & {$[39]$} \\
Liver:blood & 3.02 & {$[39]$} \\
Lung:air & 87.4 & {$[42]$} \\
\hline
\end{tabular}

is a sufficient conversion. Physiological parameters used in the PBPK model are presented in Tables II and II. As described in [46], the lung:air partition coefficient $\left(P_{\text {alv:air }}^{x y l}\right)$ was obtained from [42] and was used with the blood:air partition coefficient from [39] in order to find $P_{a l v}^{x y l}$ (i.e., $P_{\text {alv }}^{x y l}=P_{\text {alv:air }}^{x y l}\left(P_{\text {bl:air }}^{x y l}\right)$. Metabolic values from the PBPK source model are used as follows,

$$
\begin{aligned}
& V_{\max }^{x y l}=V_{\operatorname{maxc}}^{x y l} B W^{0.75} \\
& K_{m}^{x y l}=0.2,
\end{aligned}
$$

and cardiac output and alveolar ventilation are assumed to follow allometric scaling with scaling factors used from the source model

$$
Q_{c}=18.0 \cdot B W^{0.70}=Q_{p}
$$

\section{C. $V_{\operatorname{maxc}}$ values}

Metabolism of xylene is described using Michaelis-Menten kinetics in the model [39] which involves a nonlinear term with the parameter $V_{m a x}^{x y l}$ representing the maximum rate of the enzymatic process (see Equation (3)). The value of $V_{\max }^{x y l}$ is scaled to body weight as follows:

$$
V_{\max }^{x y l}=V_{\operatorname{maxc}}^{x y l} B W^{0.75} .
$$

Different values of $V_{\text {maxc }}^{x y l}$ have been used in PBPK modeling and other research as is shown in Table III] Parameter values have been rescaled for
TABLE II

THE COMPARTMENTAL VOLUMES USED IN SIMULATIONS OF THE XYLENE PBPK MODEL. AlL VALUES ARE THE SAME AS USED FOR THE "NON-AGED ADULT" IN [47] EXCEPT FOR $V_{r}, V_{b l}, V_{a l v}, V_{a r t}$, AND $V_{v e n} . V_{r}$ WAS ALTERED TO HAVE ALL VOLUMES SUM TO $100 \% . V_{b l} V_{a l v}$, $V_{\text {art }}$, AND $V_{v e n}$ ARE BASED ON [5].

\begin{tabular}{|l|r|}
\hline Volume & Value \\
\hline$V_{l}$ & $0.026 B W$ \\
$V_{f}$ & $0.214 B W$ \\
$V_{s}$ & $0.613 B W$ \\
$V_{r}$ & $0.06 B W$ \\
$V_{b l}$ & $0.079 B W$ \\
$V_{a l v}$ & $0.008 B W$ \\
$V_{\text {art }}$ & $0.2 V_{b l}$ \\
$V_{\text {ven }}$ & $0.8 V_{b l}$ \\
\hline
\end{tabular}

consistency to represent $V_{\operatorname{maxc}}^{x y l}$ as in 9). Note that the PBPK source model for the work in [46] focuses on m-xylene (as do the majority of the references in Table III). The values from [41] are estimated through conversion using different values for liver size. The values in Table III are used as a basis for $V_{\text {maxc }}^{x y l}$ values used in the simulations. $V_{\operatorname{maxc}}^{x y l}$ values used in simulations or found in optimized fits to simulated output fall within a range of $1-11 \mathrm{mg} /(\mathrm{h} \cdot \mathrm{kg})$ which is similar to the range of values presented in Table III.

\section{Investigational Methods}

Computational solutions are generated using MATLAB R2015b [27] with the ode15s solver. With the exception of values for $V_{\operatorname{maxc}}^{x y l}$, all initial conditions, exposure scenarios $(50 \mathrm{ppm}$ xylene over 7 hours), and parameters remain unchanged from the previous investigation [46].

Solution curves are generated for values of $V_{\text {maxc }}^{x y l}$ ranging from $V_{\text {maxc }_{0}}^{x y l}=1$ to $V_{\text {maxc }}^{x y l \text { final }}=$ 10 , which are chosen to illustrate the trend of output as well as include the value, 8.4, used in the original xylene model [39]. A list of $V_{\operatorname{maxc}_{i}}^{x y l}$ values is generated with spacing 0.1 , and computational solutions are generated for each metabolic constant in this list. The exposure duration is 7 hours, and 20 hours are used for output calculations to allow for the majority of the toxicant to be cleared from the system. Maximum output values over the 
K.A. Yokley, J. Ashcraft, N.S. Luke, A computational investigation of the ventilation structure and ...

TABLE III

TABLE OF VARIOUS VALUES OF $V_{\text {max }}^{x y l}$ VALUES AND REFERENCES CITING THEM. NUMERICAL VALUES HAVE BEEN RESCALED FOR CONSISTENCY. LISTED SOURCES EITHER USED THE VALUE GIVEN (NOT NECESSARILY SCALED) OR USED THE DATA FROM THE PAPER IN SOME WAY. NOTE THAT THE REFERENCE MARKED (*) CITED THE GIVEN SOURCE BUT USED 6.49 .

\begin{tabular}{|c|c|c|}
\hline Primary Source & Scaled $V_{\operatorname{maxc}}^{x y l}$ & Cited By \\
\hline $\begin{array}{l}\text { Tardif et al. } 1993[40] \text { and } \\
\text { Tardif et al. } 1995[39]\end{array}$ & $8.4 \mathrm{mg} /(\mathrm{h} \cdot \mathrm{kg})$ & $\begin{array}{l}{[12], \text { [19], [21], |22], |24], }} \\
{[31], \text { [44], [45], [46] }}\end{array}$ \\
\hline $\begin{array}{l}\text { Tardif et al. } 1997[38] \\
\text { Tassaneeyakul et al. } 1996 \text { [41] }\end{array}$ & $\begin{array}{l}5.5 \mathrm{mg} /(\mathrm{h} \cdot \mathrm{kg}) \\
\approx 11.3-13.7 \mathrm{mg} /(\mathrm{h} \cdot \mathrm{kg})\end{array}$ & $\frac{[26],[18]^{*}}{[29],[30]}$ \\
\hline Price and Krishnan 2011 [33] & $\begin{array}{l}\text { Measured: } 6.475 \mathrm{mg} /(\mathrm{h} \cdot \mathrm{kg}) \text {, } \\
\text { Predicted: } 5.300 \mathrm{mg} /(\mathrm{h} \cdot \mathrm{kg})\end{array}$ & [7], [32] \\
\hline Haddad et al. 1999 [20] & $6.59 \mathrm{mg} /(\mathrm{h} \cdot \mathrm{kg})$ & [22] \\
\hline
\end{tabular}

20 hour simulations are found for the amount of xylene in exhaled air, concentration of xylene in the venous blood, and amount of xylene in the liver. Output over the 20 hours is generated at each 0.05 step, and a Riemann sum is used to estimate area below the curve for the xylene concentration in the liver. Output in amount is converted to concentration before the area is estimated. The xylene concentration in the venous blood and amount in the exhaled air are investigated because both could be physically collected, and those data could be used to estimate $V_{\operatorname{maxc}}^{x y l}$ values. The predictions for xylene in the liver are investigated because the liver is often a target organ (in general for various toxicants) and for the use of liver predictions in risk assessment.

In order to ascertain how different $V_{\text {maxc }}^{x y l}$ values could produce similar results with the two different models, the following procedure is employed. Simulated data is generated from each model using a beginning $V_{\operatorname{maxc}}^{x y l}$ value for one model output that conceivably could be compared to measured data. Different levels of noise are added to the data, and then both models are optimized to the simulated data over $V_{\text {maxc }}^{x y l}$, minimizing a least squares cost function

$$
J\left(V_{\operatorname{maxc}}\right)=\min \sum_{i}\left(x_{i}\left(V_{\operatorname{maxc}}^{x y l}\right)^{2}-d_{i}^{2}\right)
$$

where $x_{i}$ represents the state of the differential equation model (using a particular value of $V_{\operatorname{maxc}}^{x y l}$ ) at time $t_{i}$ corresponding to data point $d_{i}$. Optimization is performed using the fminsearchbnd function [28] from the Matlab Optimization Toolbox. Data are simulated for the concentration of xylene in exhaled air and in the venous blood for both the equilibrium and non-equilibrium models and then fit to output of each model.

\section{RESUlTS}

The amount (or concentration) of a chemical within any given compartment during exposure is characterized by an increase during the interval of exposure followed by a gradual decline or clearing of the chemical after exposure has ceased. Between the interval of exposure and the clearance of the chemical, the amount of chemical in any given compartment will reach a maximum level. In order to fit chemical exposure data, it is imperative that the PBPK model under consideration can reach these maximum levels. As a premilinary exercise for this study, the maximum model outputs are generated for both the equilibrium and nonequilibrium models and compared to determine if there is any overlap.

Figure 2 depicts the relationship between maximum predicted xylene amount in the exhaled air and values of $V_{\operatorname{maxc}}^{x y l}$ for both the equlibrium and non-equlibrium models. It is evident from this graph that there is little to no overlap in the maximum predicted xylene amount for both models. 


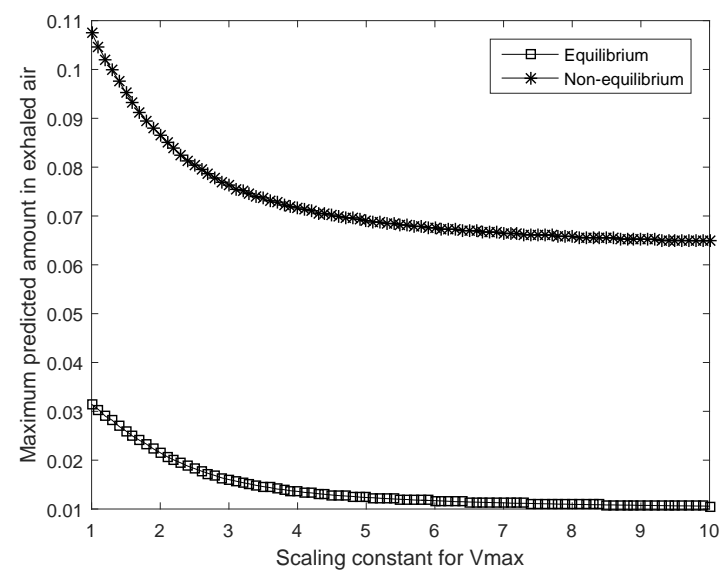

Fig. 2. Maximum model output for the amount of xylene in exhaled air for various $V_{\operatorname{maxc}}^{x y l}$ values.

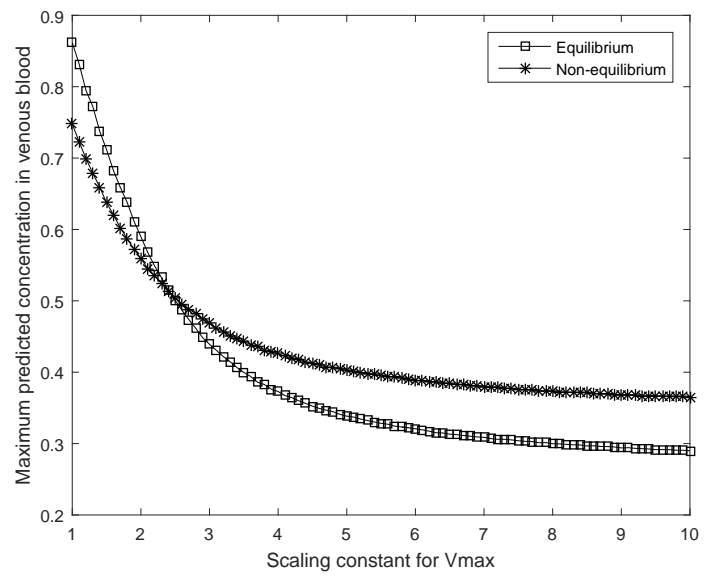

Fig. 3. Maximum model output for the concentration of xylene in the venous blood for various $V_{\operatorname{maxc}}^{x y l}$ values.

The maximum amounts generated from the nonequilibrium model are consistently higher than those of the equilibrium model. The significant difference between the model outputs would suggest that the non-equilibrium model would not be capable of fitting data generated by the equilibrium model, and the equilibrium model would not be capable of fitting data that was generated by the non-equilibrium model.

Figures 3 and 4 illustrate the relationship between values of $V_{\operatorname{maxc}}^{x y l}$ with the maximum concentration of xylene in the venous blood and maxi-

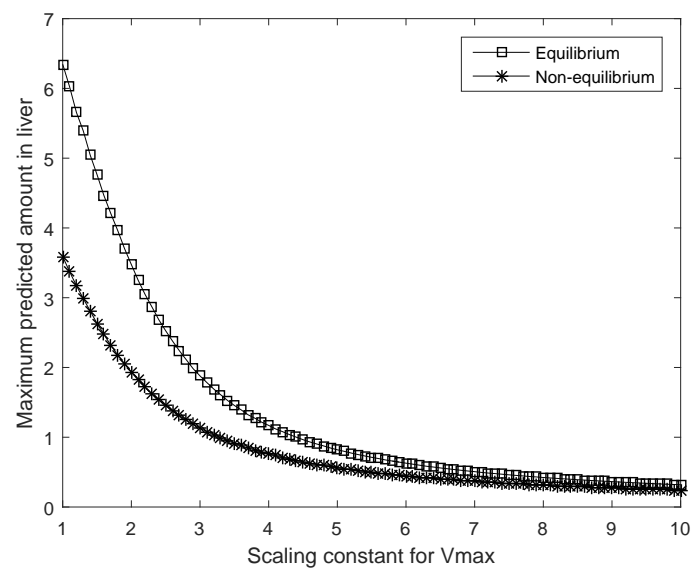

Fig. 4. Maximum model output for the amount of xylene in the liver for various $V_{\operatorname{maxc}}^{x y l}$ values.

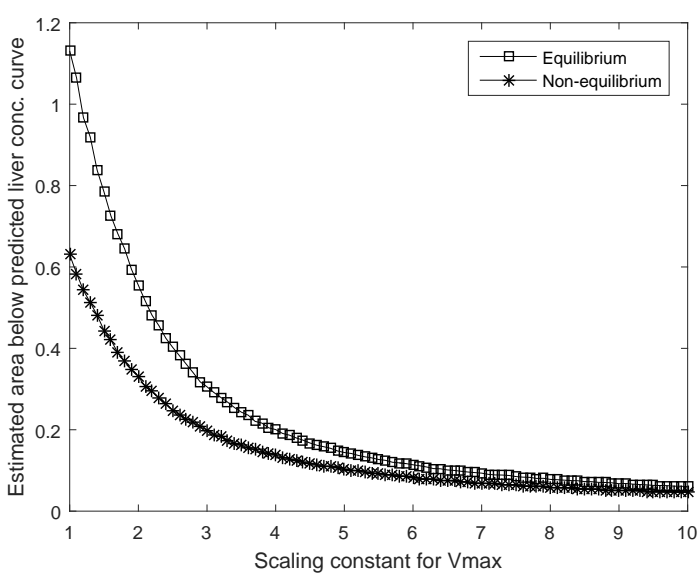

Fig. 5. Estimated area below the predicted liver concentration curve for various $V_{\operatorname{maxc}}^{x y l}$ values.

mum amount of xylene in the liver, respectively. In contrast to the maximum xylene amount in the exhaled air (Figure 2), maximum values of xylene in the venous blood and liver show a considerable amount of overlap between the equilibrium and non-equilibrium models. This indicates that the same maximum level may be predicted using either the equilibrium model or the non-equilibrium model with different values for $V_{\operatorname{maxc}}^{x y l}$. A similar relationship for the area below the curve estimates for concentation of xylene in the liver is presented in Figure 5. 


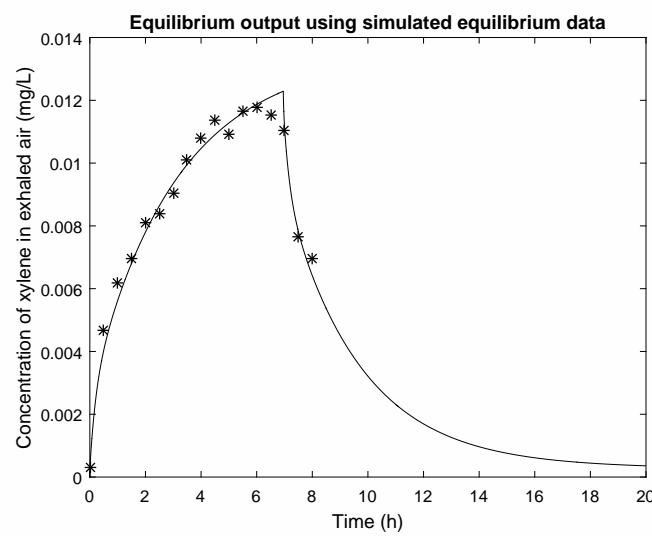

(a) Equilibrium Output

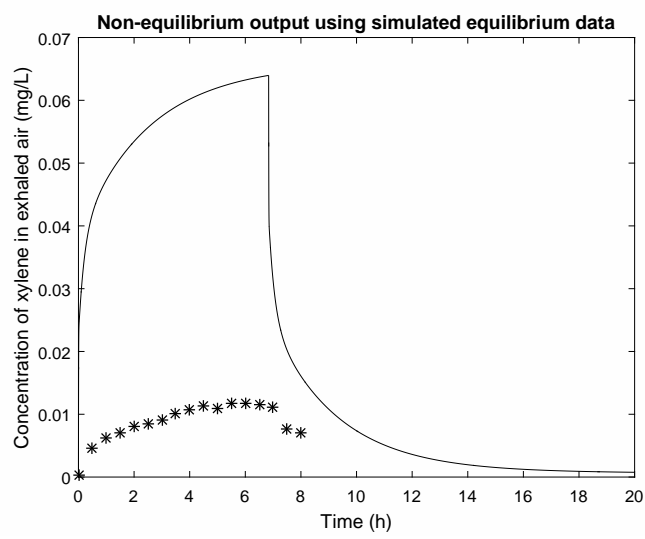

(b) Non-Equilibrium Output

Fig. 6. Model output for the concentration of xylene in exhaled air after optimization over data simulated from the equilibrium model with noise level 0.001 and $V_{\operatorname{maxc}}^{x y l}=6$. Figure (a) contains equilibrium model output and Figure (b) contains nonequilibrium model output.

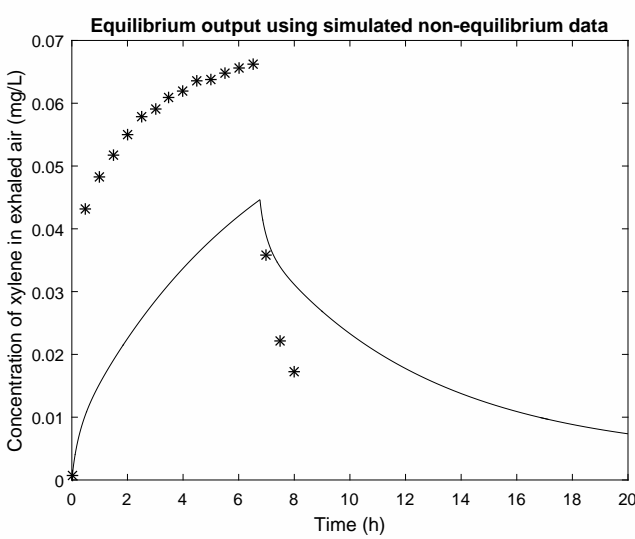

(a) Equilibrium Output

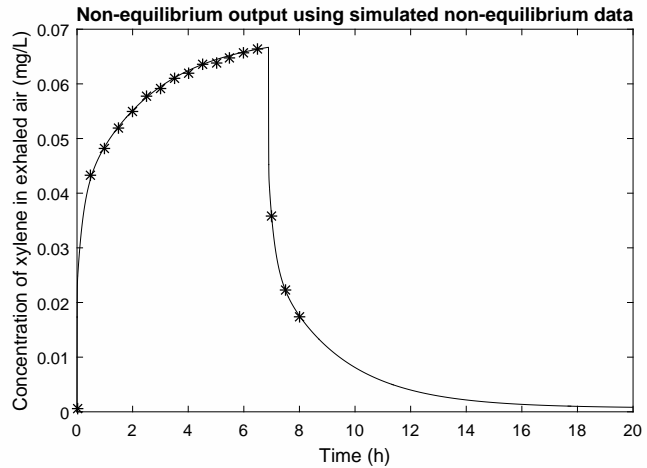

(b) Non-Equilibrium Output

Fig. 7. Model output for the concentration of xylene in exhaled air after optimization over data simulated from the nonequilibrium model with noise level 0.001 and $V_{\operatorname{maxc}}^{x y l}=8$. Figure (a) contains equilibrium model output and Figure (b) contains non-equilibrium model output.

Based on these comparisons of the maximum value of xylene for each variation of the model, it seems that the equilibrium and non-equilibrium models would fit the opposite data set more efficiently for the concentration of xylene in the venous blood and the amount of xylene in the liver than they would for the amount of xylene in the exhaled air. While the liver is a target organ for risk assessment, experimental data for the amount of a chemical within the liver is not easily collected; thus it is often unavailable. For these reasons, more focus is placed on predictions of blood concentrations for this study.

Examples of graphical output for xylene in exhaled air using simulated data from the equilibrium model are contained in Figure 6, and examples using simulated data from the non-equilibrium model are contained in Figure 7. These figures provide examples of best fitting curves to the simulated data. A summary of simulation results for exhaled 
K.A. Yokley, J. Ashcraft, N.S. Luke, A computational investigation of the ventilation structure and ...

TABLE IV

OPTIMAL COST USING SIMULATED DATA FOR THE CONCENTRATION OF XYLENE IN EXHALED AIR. THE $V_{\text {maxc }}^{x y}$ VALUE USED FOR SIMULATION IS LISTED IN COLUMN 1 OF THE TABLE, AND INITIAL GUESSES FOR $V_{\text {maxc }}^{x y l}$ FOR THE OPTIMIZATION ROUTINE WERE 3,6 , AND 10 . THE VALUE FOR $J^{*}$ THAT WAS LOWEST FOR THE THREE IS LISTED BELOW. IN CASES MARKED WITH $(\dagger)$ THE SAME COST WAS FOUND FOR VARIOUS $V_{\text {maxc }}^{x y l}$ VALUES, ALL OF WHICH WERE CLOSE TO ZERO.

\begin{tabular}{|c|c|c|c|}
\hline \multicolumn{4}{|c|}{ Equilibrium simulated data } \\
\hline$V_{\operatorname{maxc}}^{x y l}$ & Noise $\|$ & Best Fit to "Equilibrium" & Best Fit to "Non-equilibrium" \\
\hline $\begin{array}{l}2 \\
4 \\
6\end{array}$ & $\begin{array}{l}0.001 \\
0.001 \\
0.001\end{array}$ & $\begin{array}{l}V_{\operatorname{maxc}}^{x y l}=1.9125, J^{*}=1.6885 e^{-6} \\
V_{\operatorname{maxc}}^{x y l}=3.7144, J^{*}=1.8447 e^{-6} \\
V_{\operatorname{maxc}}^{x y l}=5.1232, J^{*}=2.1612 e^{-6}\end{array}$ & $\begin{array}{l}V_{\operatorname{maxc}}^{x y l}=14.9393, J^{*}=0.0224 \\
V_{m a x c}^{x y l}=14.9386, J^{*}=0.0281 \\
V_{\operatorname{maxc}}^{x y l}=14.9383, J^{*}=0.0299\end{array}$ \\
\hline \multicolumn{4}{|c|}{ Non-equilibrium simulated data } \\
\hline$V_{\operatorname{maxc}}^{x y l}$ & Noise $\|$ & Fit to "Equilibrium" & Fit to "Non-equilibrium" \\
\hline $\begin{array}{l}4 \\
6 \\
8\end{array}$ & $\begin{array}{l}0.001 \\
0.001 \\
0.001\end{array}$ & $\begin{array}{l}V_{\operatorname{maxc}}^{x y l}=0.0014^{\dagger}, J^{*}=0.0151 \\
V_{T \text { ax }}^{x y l}=0.0013^{\dagger}, J^{*}=0.0123 \\
V_{\operatorname{maxc}}^{\text {xyl }}=0.0051^{\dagger}, J^{*}=0.0114\end{array}$ & $\begin{array}{l}V_{\operatorname{maxc}}^{x y l}=3.5138, J^{*}=4.9275 e^{-5} \\
V_{m a x c}^{x y l}=5.7957, J^{*}=4.3432 e^{-6} \\
V_{\operatorname{maxc}}^{x y l}=7.0188, J^{*}=1.5291 e^{-6}\end{array}$ \\
\hline
\end{tabular}

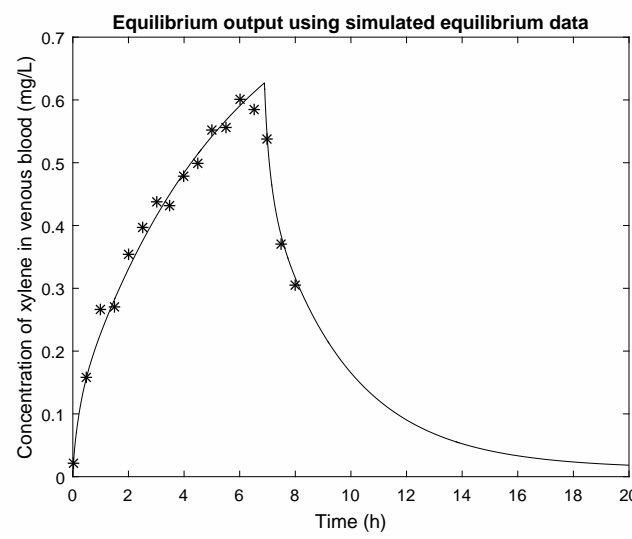

(a) Equilibrium Output

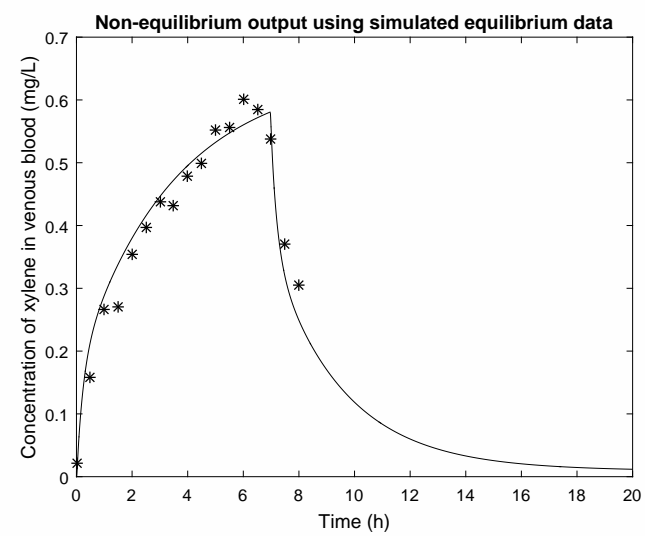

(b) Non-Equilibrium Output

Fig. 8. Model output for the concentration of xylene in venous blood after optimization over data simulated from the equilibrium model with noise level 0.05 and $V_{\operatorname{maxc}}^{x y l}=2$. Figure (a) contains equilibrium model output and Figure (b) contains non-equilibrium model output.

air concentration is presented in Table IV

In Figure 6, it can be observed that the equilibrium model (depicted in Figure 6(a) provides a much better fit to the equilibrium data than the non-equilibrium model (depicted in Figure 6(b)p does. When fit to the equilibrium data, the equilibrium model produces a least squares cost of $2.1612 e^{-6}$ and the non-equilibrium model produces a cost of 0.0299. Similary, Figure 7 shows that the non-equilibrium model provides a much better fit to the non-equilibrium data than that of the equilibrium model. The non-equilibrium model yields a least squares cost of $1.5291 e^{-6}$ with the non-equilbrium data, compared to the equilibrium model's cost of 0.0114 .

Data are also simulated for the concentration of xylene in venous blood for both the equilibrium and non-equilibrium models. The fits to these models show some success for $V_{\operatorname{maxc}}^{x y l}$ in the range of 3-7. Examples of graphical results are contained 


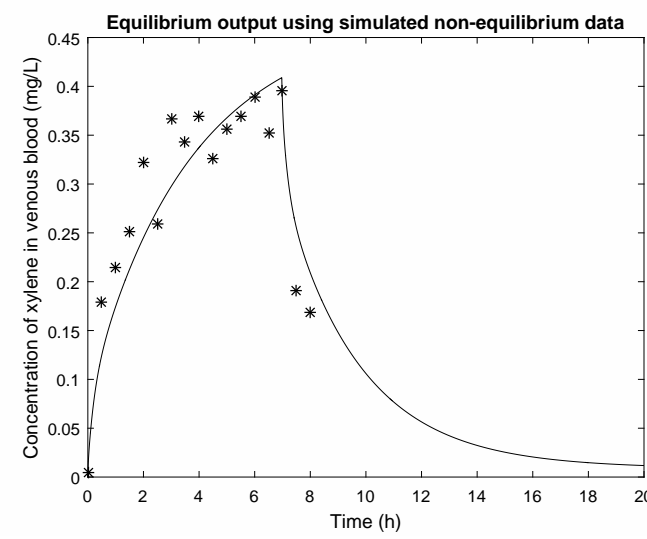

(a) Equilibrium Output, noise $=0.02$

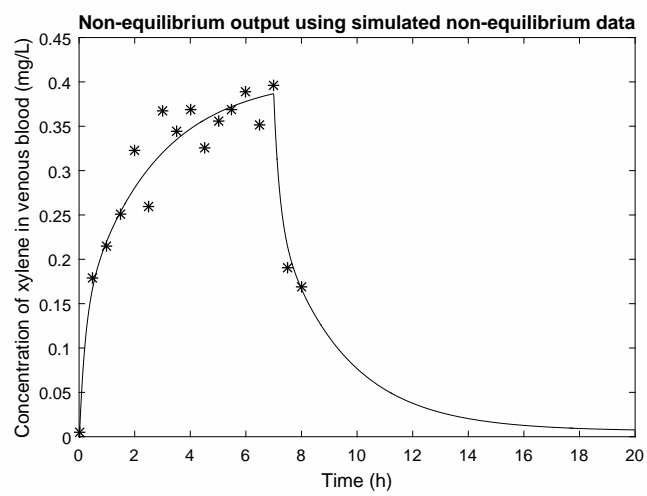

(c) Non-Equilibrium Output, noise $=0.02$

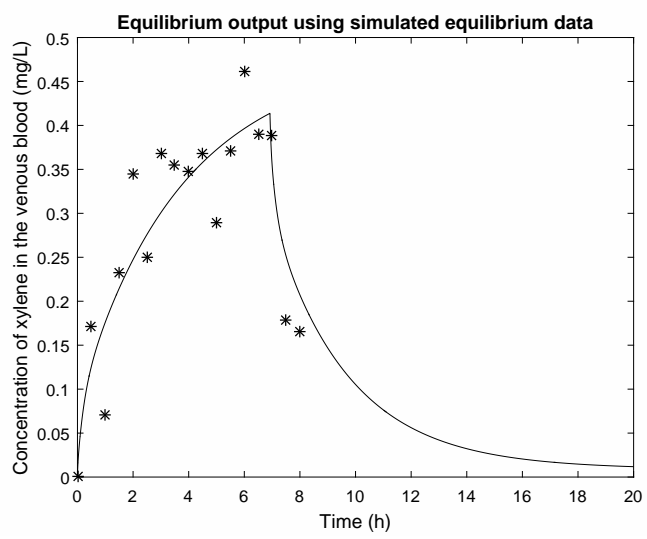

(b) Equilibrium Output, noise $=0.05$

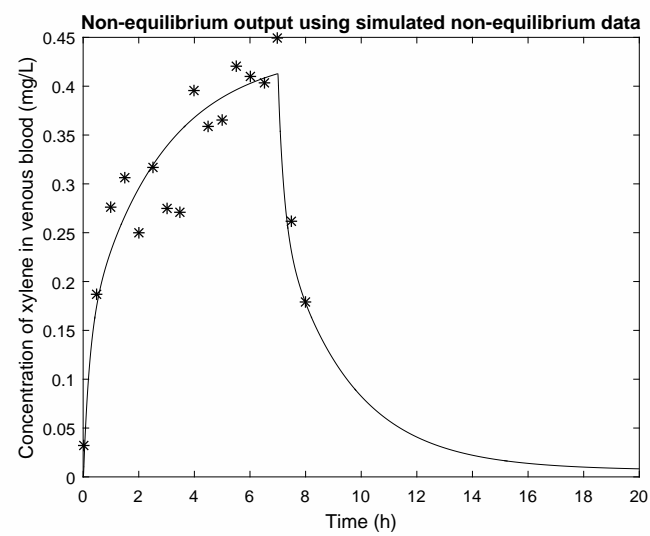

(d) Non-Equilibrium Output, noise $=0.05$

Fig. 9. Model output for the concentration of xylene in venous blood after optimization over data simulated from the non-equilibrium model with given noise level and $V_{\operatorname{maxc}}^{x y l}=6$. Figures (a)-(b) contain equilibrium model output and Figures (c)-(d) contain non-equilibrium model output.

in Figure 8 and Figure 9, and a summary of overall results for the concentration of xylene in venous blood is presented in Table $\mathrm{V}$.

Figure 8 illustrates the best fits of the equilibrium and non-equilibrium models to venous blood concentration data that was generated by the equilibrium model with a $V_{\operatorname{maxc}}^{x y l}$ value of 2 . Unlike the previously presented results for the model fits to exhaled air, both models seem to provide an adequate fit to the venous blood data. The results from the equilibrium model (in Figure $8(\mathrm{a})$ seem to capture the maximum more efficiently. The best fit for the equilibrium model is produced using a $V_{\operatorname{maxc}}^{x y l}$ value of 1.8299 , with a cost function value of 0.0058 . The best fit for the non-equilibrium model is produced with a $V_{\operatorname{maxc}}^{x y l}$ value of 1.8514 , yielding a cost of 0.0203 .

The fits of the equilibrium and non-equilibrium models to non-equilibrium data are presented in Figure 9. Figures 9(a) and 9(b) display the fit of the equilibrium model to non-equilibrium data with noise levels of $2 \%$ and $5 \%$, respectively. Results for the non-equilibrium model fitted to the nonequilibrium data with noise levels of $2 \%$ and $5 \%$ are depicted in Figures 9(c) and 9(d). A visual inspection of the graphs would suggest that both the equilibrium model and the non-equilibrium model can provide an adequate fit to the simulated 
K.A. Yokley, J. Ashcraft, N.S. Luke, A computational investigation of the ventilation structure and ...

TABLE V

OPTIMAL COST USING SIMULATED DATA FOR THE CONCENTRATION OF XYLENE IN VENOUS BLOOD. THE $V_{\text {maxc }}^{x y l}$ VALUE USED FOR SIMULATION IS LISTED IN COLUMN 1 OF THE TABLE, AND INITIAL GUESSES FOR $V_{\text {maxc }}^{x y l}$ FOR THE

OPTIMIZATION ROUTINE WERE 3, 6, AND 10. THE VALUE FOR $J^{*}$ THAT WAS LOWEST FOR THE THREE IS LISTED BELOW.

\begin{tabular}{|c|c|c|c|}
\hline \multicolumn{4}{|c|}{ Equilibrium simulated data } \\
\hline$V_{\operatorname{maxc}}^{x y l}$ & Noise & Best Fit to "Equilibrium" & Best Fit to "Non-equilibrium" \\
\hline 2 & 0.001 & $V_{\operatorname{maxc}}^{x y l}=1.9687, J^{*}=2.2307 e^{-4}$ & $V_{\operatorname{maxc}}^{x y l}=2.0752, J^{*}=0.0247$ \\
\hline 2 & 0.01 & $V_{\operatorname{maxc}}^{x y l}=1.9596, J^{*}=4.7682 e^{-4}$ & $V_{\operatorname{maxc}}^{x y l}=2.0700, J^{*}=0.0234$ \\
\hline 2 & 0.02 & $V_{\operatorname{maxc}}^{x y l}=1.9173, J^{*}=7.6803 e^{-4}$ & $V_{\operatorname{maxc}}^{x y l}=2.0190, J^{*}=0.0237$ \\
\hline 2 & 0.05 & $V_{\operatorname{maxc}}^{x y l}=1.8299, J^{*}=0.0058$ & $V_{\operatorname{maxc}}^{x y l}=1.8514, J^{*}=0.0203$ \\
\hline 3 & 0.001 & $V_{\operatorname{maxc}}^{x y l}=3.0000, J^{*}=7.1896 e^{-6}$ & $V_{\operatorname{maxc}}^{x y l}=4.8376, J^{*}=0.0139$ \\
\hline 3 & 0.01 & $V_{\operatorname{maxc}}^{x y l}=2.9006, J^{*}=2.3456 e^{-4}$ & $V_{\operatorname{maxc}}^{x y l}=4.5075, J^{*}=0.0132$ \\
\hline 3.5 & 0.05 & $V_{\operatorname{maxc}}^{x y l}=3.1610, J^{*}=0.0058$ & $V_{\operatorname{maxc}}^{x y l}=5.4462, J^{*}=0.0127$ \\
\hline \multicolumn{4}{|c|}{ Non-equilibrium simulated data } \\
\hline$V_{\operatorname{maxc}}^{x y l}$ & Noise & Fit to "Equilibrium" & Fit to "Non-equilibrium" \\
\hline 6 & 0.001 & $V_{\operatorname{maxc}}^{x y l}=3.3016, J^{*}=0.0128$ & $V_{\operatorname{maxc}}^{x y l}=6.0000, J^{*}=8.5450 e^{-6}$ \\
\hline 6 & 0.01 & $V_{\operatorname{maxc}}^{x y l}=3.3067, J^{*}=0.0154$ & $V_{\operatorname{maxc}}^{x y l}=6.2866, J^{*}=7.8847 e^{-4}$ \\
\hline 6 & 0.02 & $V_{\operatorname{maxc}}^{x y l}=3.3163, J^{*}=0.0201$ & $V_{\operatorname{maxc}}^{x y l}=6.6882, J^{*}=0.0050$ \\
\hline 6 & 0.05 & $V_{\operatorname{maxc}}^{x y l}=3.3067, J^{*}=0.0489$ & $V_{\operatorname{maxc}}^{x y l}=6.2866, J^{*}=0.0466$ \\
\hline 8 & 0.001 & $V_{\operatorname{maxc}}^{x y l}=3.6121, J^{*}=0.0111$ & $V_{\operatorname{maxc}}^{x y l}=10.6876, J^{*}=8.9750 e^{-4}$ \\
\hline 8 & 0.01 & $V_{\operatorname{maxc}}^{x y l}=3.5750, J^{*}=0.0111$ & $V_{\operatorname{maxc}}^{x y l}=10.2790, J^{*}=0.0017$ \\
\hline
\end{tabular}

non-equilibrium data. The non-equilibrium data in these graphs were generated with a $V_{\operatorname{maxc}}^{x y l}$ value of 6 . The best fit of the equilibrium model to the data with $2 \%$ noise is found with $V_{\text {maxc }}^{x y l}$ equal to 3.3163 and has a least squares cost of 0.0201 . A $V_{\operatorname{maxc}}^{x y l}$ value of 3.3067 leads to the optimal fit of the equilibrium model to the non-equilibrium data with $5 \%$ noise, resulting in a cost of 0.0489 . The best fits for the non-equilibrium model to the non-equilibrium data with $2 \%$ and $5 \%$ noise are produced with $V_{\operatorname{maxc}}^{x y l}$ values of 6.6882 and 6.2866 and result in cost values of 0.0050 and 0.0466 , respectively.

As previously stated, the amount of xylene present in the liver following an inhalation exposure is not easily measured and therefore not reported. For this reason, a comparison of the equilibrium and non-equilibrium model results for the amount of xylene in the liver is not conducted. Based on the maximum model output for the amount of xylene in the liver (depicted in Figure 4), it is hypothesized that adequate fits to data can be found using both the equilibrium and non- equilibrium models (similar to the results reported for the venous blood concentrations above).

\section{Discussion AND CONCLUSIONS}

Simulations suggest that different $V_{\operatorname{maxc}}^{x y l}$ values could be used to make similar predictions for the concentration of xylene in venous blood with the different ventilation structures, but different $V_{\operatorname{maxc}}^{x y l}$ values are not found to produce similar model output for the amount in exhaled air. Specifically, using $V_{\operatorname{maxc}}^{x y l}$ around 3 in the "equilibrium" model produces similar blood concentration results as using $V_{\operatorname{maxc}}^{x y l}$ around 6 in the "non-equilibrium" model. As shown in Figure 3 and in Figure 9, the maximum xylene concentration is predicted to be about $0.4 \mathrm{mg} / \mathrm{L}$ by both "equilibrium" and "non-equilibrium" models when $V_{\operatorname{maxc}}^{x y l}$ is around 3 or 6 , respectively. Additionally, optimization to simulated data results in best fits with similar numbers (see results in Table $\mathrm{V}$ for simulated $V_{\operatorname{maxc}}^{x y l}=3$, 3.6, and 6). Hence, blood data may not be as informative as exhaled air data for identifying the maximum rate of metabolism when 
K.A. Yokley, J. Ashcraft, N.S. Luke, A computational investigation of the ventilation structure and ...

the exposure method is inhalation. Results may differ if a toxicant is administered in the blood directly or ingested or exposed dermally as in [36]. In cases involving exposure through methods other than inhalation, the ventilation equation structure would be expected to be less critical and so are not investigated in the current study. However, in those cases, the equations describing the entrance of toxicant into the body could be critical as well.

Figures 4 and 5 contain predictions related to xylene in the liver. When these predictions are calculated using the "equilibrium" model with $V_{\operatorname{maxc}}^{x y l}$ around 3 and the "non-equilibrium" model with $V_{\text {maxc }}^{x y l}$ around 6, however, the liver predictions are higher for the "equilibrium" model. Although the "equilibrium" model for the xylene PBPK model used here may then provide higher predictions for internal liver doses, the "equilibrium" ventilation structure may not provide higher internal dose estimates when used with more complicated PBPK models. For example, the results in Figures 4 and 5 contain predictions for the parent chemical, and concentrations of metabolites may show different dynamics.

Although the PBPK models of xylene used in the current study do not focus on the excretion of toxicants, some models do make predictions of the parent chemical or metabolites in the urine. Data of excreted toxicants could also be problematic for use in optimizing metabolic parameters. Additionally, the model used in the current study is a more simplistic PBPK model, and more investigation is needed to be able to make conclusions about toxicants with harmful metabolites or that require models with more compartments. PBPK models have been used to describe exposure to a mixture of chemicals (such as in [12] [18] [24] [33][39] [43]) which would also involve a more complicated investigation than in the current study.

\section{AcKnowledgements}

The authors would like to thank Elon University Funding for Research and Development for support for this project.

\section{APPENDIX}

Abbreviations:

$\begin{array}{cl}x y l & \text { xylene } \\ s & \text { slowly perfused tissues } \\ f & \text { adipose tissue or fat } \\ r & \text { rapidly perfused tissues } \\ l & \text { liver } \\ \text { ven } & \text { venous blood } \\ \text { art } & \text { arterial blood } \\ b l & \text { blood (mixed) } \\ \text { alv } & \text { alveolar space or respiratory } \\ & \text { compartment }\end{array}$

Model Notations:

$Q_{j} \quad$ Blood flow in/to compartment $j(L / h)$

$Q_{c} \quad$ Cardiac flow, $\sum_{j} Q_{j}(L / h)$

$Q_{p} \quad$ Pulmonary flow/alveolar ventilation $(L / h)$

$V_{j} \quad$ Volume of compartment $j(L)$

$P_{j}^{i} \quad$ The partition coefficient for chemical $i$ in tissue $j$ (dimensionless)

$P_{b l: a i r}^{x y l} \quad$ The blood/air partition coefficient for chemical xyl (dimensionless)

$A_{j}^{x y l} \quad$ The amount of chemical $x y l$ in tissue $j(m g)$

$C_{j}^{x y l} \quad$ The concentration of chemical $x y l$ in compartment $j(m g / L)$

$C_{i n h}^{x y l} \quad$ The concentration of chemical $x y l$ inhaled $(m g / L)$

$V_{\max }^{x y l} \quad$ The maximum rate of metabolism for chemical $x y l(m g / h)$

$K_{m}^{x y l} \quad$ The concentration of $x y l$ at half saturation $(m g / L)$

\section{REFERENCES}

[1] Abbas, R, Fisher, JW (1997) A Physiologically Based Pharmacokinetic Model for Trichloroethylene and Its Metabolites, Chloral Hydrate, Trichloroacetate, Dichloroacetate, Trichloroethanol, and Trichloroethanol 
Glucuronide in $\mathrm{B}_{6} \mathrm{C} 3 \mathrm{~F}_{1}$ Mice, Toxicol Appl Pharm, 147:15-30.

[2] Allen, BC, Fisher, JW (1993) Pharmacokinetic Modeling of Trichloroethylene and Trichloroacetic Acid in Humans, Risk Anal, 13(1):71-86.

[3] Blancato, JN, Evans, MV, Power, FW, Caldwell, JC (2007) Development and Use of PBPK Modeling and the Impact of Metabolism on Variability in Dose Metrics for the Risk Assessment of Methyl Tertiary Butyl Ether (MTBE), Journal of Environmental Protection Science, $1: 29-51$.

[4] Brautbar, N, Williams, J (2002) Industrial liver toxicity: Risk assessment, risk factors and mechanisms, Int J Hyg Envir Heal 205(6):479-491.

[5] Brown, RP, Delp, MD, Lindstedt, SL, Rhomberg, LR, Beliles, RP (1997) Physiological Parameter Values for Physiologically Based Pharmacokinetic Models. Toxicol Ind Health 13(4):407-484.

[6] Caldwell, JC, Evans, MV, Krishnan, K (2012) Cutting Edge PBPK Models and Analyses: Providing the Basis for Future Modeling Efforts and Bridges to Emerging Toxicology Paradigms, Journal of Toxicology 2012: 852384 (doi:10.1155/2012/852384).

[7] Cheng, S, Bois, FY (2011) A mechanistic modeling framework for predicting metabolic interactions in complex mixtures. Environ Health Persp 119(12):17121718.

[8] Clewell, HJ, Gentry, PR, Covington, TR, Gearhart, JM (2000) Development of a Physiologically Based Pharmacokinetic Model of Trichloroethylene and Its Metabolites for Use in Risk Assessment, Environ Health Persp 108(Suppl 2):283-305.

[9] Cole, CE, Tran, HT, Schlosser, PM (2001) Physiologically Based Pharmacokinetic Modeling of Benzene Metabolism in Mice Through Extrapolation from In Vitro to In Vivo, J Toxicol Env Heal A 62(6):439-465.

[10] Csanády, GA, Kessler, W, Hoffmann, HD, Filser, JG (2003) A toxicokinetic model for styrene and its metabolite styrene-7,8-oxide in mouse, rat and human with special emphasis on the lung, Toxicol Lett 138(12):75-102

[11] Cuello, WS, James, TAT, Jessee, JM, Venecek, MA, Sawyer, ME, Eklund, CR, Evans, MV (2012) Physiologically Based Pharmacokinetic (PBPK) Modeling of Metabolic Pathways of Bromochloromethane in Rats, Journal of Toxicology 2012: 629781 (doi: 10.1155/2012/629781).

[12] Dobrev, ID, Andersen, ME, Yang, RSH (2002) In silico toxicology: simulating interaction thresholds for human exposure to mixtures of trichloroethylene, tetrachloroethylene, and 1, 1, 1-trichloroethane. Environ Health Persp 110(10):1031-1039.

[13] El-Masri, HA, Portier, C (1198) Physiologically Based Pharmacokinetics Model of Primidone and Its Metabolites Phenobarbital and Phenylethylmalonamide in $\mathrm{Hu}-$ mans, Rats, and Mice, Drug Metab Dispos 26(6):585594.
[14] Evans, MV, Crank, WD, Yang, H, Simmons, JE (1994) Applications of a sensitivity analysis to a physiologically based pharmacokinetic model for carbon tetrachloride in rats. Toxicol Appl Pharm 128:36-44.

[15] Fisher, JW, Mahle, D, Abbas, R (1998) A Human Physiologically Based Pharmacokinetic Model for Trichloroethylene and Its Metabolites, Trichloroacetic Acid and Free Trichloroethanol, Toxicol Appl Pharm 152:339-359.

[16] Gagnaire, F, Langlais, C, Grossman, S, Wild, P (2007) Ototoxicity in rats exposed to ethylbenzene and to two technical xylene vapours for 13 weeks, Arch Toxicol 81(2):127-143.

[17] Greenberg, MS, Burton, GA, Jr., Fisher, JW (1999) Physiologically Based Pharmacokinetic Modeling of Inhaled Trichloroethylene and Its Oxidative Metabolites in $\mathrm{B}_{6} 3 \mathrm{~F}_{1}$ Mice, Toxicol Appl Pharm, 154:264-278.

[18] Haddad, S, Beliveau, M, Tardif, R, Krishnan, K (2001) A PBPK Modeling-Based Approach to Account for Interactions in the Health Risk Assessment of Chemical Mixtures, Toxicol Sci 63(1):125-131.

[19] Haddad, S, Krishnan, K (1998) Physiological modeling of toxicokinetic interactions: implications for mixture risk assessment. Environ Health Persp 106(Suppl 6):1377-1384.

[20] Haddad, S, Tardif, Charest-Tardif, G, Krishnan, K (1999) Physiological modeling of the toxicokinetic interactions in a quaternary mixture of aromatic hydrocarbons. Toxicol Appl Pharm 161(3):249-257.

[21] Krishnan, K, Clewell, HJ 3rd, Andersen, ME (1994) Physiologically based pharmacokinetic analyses of simple mixtures. Environ Health Persp 102(Suppl 9):151155.

[22] Krishnan, K, Haddad, S, Béliveau, M, Tardif, R. (2002) Physiological modeling and extrapolation of pharmacokinetic interactions from binary to more complex chemical mixtures. Environ Health Persp 110(Suppl 6):989-994.

[23] Loizou, GD (2001) The application of physiologically based pharmacokinetic modelling in the analysis of occupational exposure to perchloroethylene. Toxicol Lett 124:59-69.

[24] Loizou, GD, McNally, K, Jones, K, Cocker, J (2015) The application of global sensitivity analysis in the development of a physiologically based pharmacokinetic model for m-xylene and ethanol co-exposure in humans. Frontiers in Pharmacology 6(135):1-19.

[25] Manning, CC, Schlosser, PM, Tran, HT (2010) A Multicompartment Liver-based Pharmacokinetic Model for Benzene and its Metabolites in Mice, B Math Biol, 72(3):507-540.

[26] Marchand, A, Aranda-Rodriguez, R, Tardif, R, Nong, A, Haddad, S (2015) Human Inhalation Exposures to Toluene, Ethylbenzene, and M-Xylene and Physiologically Based Pharmacokinetic Modeling of Exposure Biomarkers in Exhaled Air, Blood, and Urine. Toxicol Sci 144(2):414-424. 
[27] Matlab, http://www.mathworks.com/

[28] fminsearch.m, Matlab Central File Exchange, http://www.mathworks.com/matlabcentral/fileexchange/ 8277-fminsearchbnd--fminsearchcon/

[29] McNally, K, Cotton, R, Cocker, J, Jones, K, Bartels, M, Rick, D, Price, P, Loizou, G (2012) Reconstruction of exposure to $\mathrm{m}$-Xylene from human biomonitoring data using PBPK modelling, Bayesian inference, and Markov chain Monte Carlo simulation. Journal of Toxicology 2012 (doi:10.1155/2012/760281).

[30] McNally, K, Cotton, R, Loizou, GD (2011) A workflow for global sensitivity analysis of PBPK models. Frontiers in pharmacology 2(31):1-22.

[31] Mendoza-Cantú, A, Castorena-Torres, F, Bermúdez de León, M, Cisneros, B, López-Carrillo, L, Rojas-García, AE, Aguilar-Salinas, A, Manno, M, Albores, A (2006) Occupational toluene exposure induces cytochrome P450 2E1 mRNA expression in peripheral lymphocytes. Environ Health Persp 114(4):494-499.

[32] Peyret, T, Krishnan, K (2012) Quantitative propertyproperty relationship for screening-level prediction of intrinsic clearance of volatile organic chemicals in rats and its integration within PBPK Models to predict inhalation pharmacokinetics in humans. Journal of Toxicology 2012 (http://dx.doi.org/10.1155/2012/286079).

[33] Price, K, Krishnan, K (2011) An integrated QSARPBPK modelling approach for predicting the inhalation toxicokinetics of mixtures of volatile organic chemicals in the rat. SAR QSAR Environ Res 22(1-2):107-128.

[34] Ramsey, JC, Andersen, ME (1984) A physiologically based description of the inhalation pharmacokinetics of styrene in rats and humans, Toxicol Appl Pharm, 73(1):159-175.

[35] Sarangapani, R, Teeguarden, JG, Cruzan, G, Clewell, HJ, Andersen, ME (2002) Physiologically based pharmacokinetic modeling of styrene and styrene oxide respiratory-tract dosimetry in rodents and humans, Inhal Toxicol 14(8):789-834.

[36] Sawyer, ME, Evans, MV, Wilson, CA, Beesley, LJ, Leon, LS, Eklund, CR, Croom, EL, Pegram, RA (2016) Development of a human physiologically based pharmacokinetic (PBPK) model for dermal permeability for lindane, Toxicol Lett 245: 106-109.

[37] Simmons, JE, Boyes, WK, Bushnel, PJl, Raymer, JH, Limsakun, T, McDonald, A, Sey, YM, Evans, MV (2002) A Physiologically Based Pharmacokinetic Model for Trichloroethylene in the Male Long-Evans Rat, Toxicol Sci 69:3-15.

[38] Tardif, R, Charest-Tardif, G, Brodeur, J, Krishnan, K (1997) Physiologically based pharmacokinetic modeling of a ternary mixture of alkyl benzenes in rats and humans. Toxicol Appl Pharm 144(1):120-134.

[39] Tardif, R, Laparé, S, Charest-Tardif, G, Brodeur, J, Krishnan, K (1995) Physiologically-based pharmacokinetic modeling of a mixture of toluene and xylene in humans. Risk Anal 15(3):335-342.

[40] Tardif, R, Laparé, S, Krishnan, K, Brodeur, J (1993) Physiologically based modeling of the toxicokinetic interaction between toluene and m-xylene in the rat. Toxicol Appl Pharm 120(2):266-73

[41] Tassaneeyakul, W, Birkett, DJ, Edwards, JW, Veronese, ME, Tassaneeyakul, W, Tukey, RH, Miners, JO (1996) Human cytochrome $\mathrm{P} 450$ isoform specificity in the regioselective metabolism of toluene and o-, m-and pxylene. J Pharmacol Exp Ther 276(1):101-108.

[42] Thrall, KD, Gies, RA, Muniz, J, Woodstock, AD, Higgins, G (2002) Route-of-entry and brain tissue partition coefficients for common superfund contaminants. J Toxicol Env Heal A 65:2075-2086.

[43] Valcke, M, Haddad, S (2015) Assessing human variability in kinetics for exposures to multiple environmental chemicals: a physiologically based pharmacokinetic modeling case study with dichloromethane, benzene, toluene, ethylbenzene, and m-xylene, J Toxicol Env Heal A 78: 409-431.

[44] Yang, RS (1998) Some critical issues and concerns related to research advances on toxicology of chemical mixtures. Environ Health Persp 106(Suppl 4):10591063.

[45] Yang, RS, Thomas, RS, Gustafson, DL, Campain, J, Benjamin, SA, Verharr, HJ, M.M. Mumtaz, MM (1998) Approaches to developing alternative and predictive toxicology based on PBPK/PD and QSAR modeling. Environ Health Persp 106(Suppl 6):1385-1393.

[46] Yokley, KA (2013) Investigations on Ventilation Equation Structure in Physiologically Based Pharmacokinetic (PBPK) Modeling of Inhaled Toxicants. International Journal of Pure and Applied Mathematics 82(1):95-123.

[47] Yokley, KA, Evans, MV (2008) Physiological changes associated with aging result in lower internal doses of toluene and perchloroethylene in simulations using pharmacokinetic modeling. Toxicol Environ Chem 90(3):75492.

[48] Yokley, KA, Evans, MV (2007) An Example of Model Structure Differences Using Sensitivity Analyses in Physiologically Based Pharmacokinetic Models of Trichloroethylene in Humans, B Math Biol 69(8):25912625. 